\title{
Comparative Study of Incretin Versus Non- Incretin Based Therapies on Type 2 Diabetes Patients in Addis Ababa, Ethiopia
}

\author{
Alias Tesfamariam ${ }^{1}$, Menakath Menon $^{1}$, Tedla Kebede ${ }^{2}$, Solomon Genet ${ }^{1,}$ * \\ ${ }^{1}$ Department of Medical Biochemistry, Addis Ababa University, Addis Ababa, Ethiopia \\ ${ }^{2}$ Department of Internal Medicine, Addis Ababa University, Addis Ababa, Ethiopia \\ Email address: \\ aliast06@gmail.com(A. Tesfamariam),menakathmenon@yahoo.com(M. Menon),tedlakgb@yahoo.com(T. Kebede), \\ solgen73@yahoo.com(S. Genet) \\ ${ }^{*}$ Corresponding author
}

\section{To cite this article:}

Alias Tesfamariam, Menakath Menon, Tedla Kebede, Solomon Genet. Comparative Study of Incretin Versus Non- Incretin Based Therapies on Type 2 Diabetes Patients in Addis Ababa, Ethiopia. Advances in Biochemistry. Vol. 6, No. 2, 2018, pp. 10-18.

doi: $10.11648 /$ j.ab.20180602.11

Received: March 24, 2018; Accepted: May 19, 2018; Published: June 11, 2018

\begin{abstract}
Type 2 diabetes is characterized by hyperglycemia due to defects in insulin secretion or insulin action. Over the years, attempts to regulate diabetics therapeutically through glycemic control have reduced complications and trauma associated with the disease. Aim of this study was to compare efficacy of incretin-based versus non-incretin based therapies on type-2 diabetic patients and a hospital based cross-sectional comparative study was conducted. Sixty type-2 diabetic patients who received non-incretin and thirty who received incretin based therapies were randomly selected for the study. The mean total cholesterol, triglyceride, low density lipoprotein, serum urea and serum creatinine were significantly lower in patients who received incretin-based therapies than those who received non-incretin based therapies. The mean high density lipoprotein was also significantly increased among the incretin based treated group. The mean body mass index and fasting blood sugar were lower in the incretin-based compared to non-incretin based treated patients. Female had insignificantly higher level of blood sugar, triglyceride, low density lipoprotein, serum urea, body mass index and lower level of total cholesterol, high density lipoprotein and serum creatinine compared to male in both groups. Incretin-based therapy helps normalize lipid profiles, blood sugar level, body mass index and also improves kidney clearance pattern.
\end{abstract}

Keywords: Type-2 Diabetes, Lipid Profile, Renal Function Test, Incretin-Based Therapy, BMI

\section{Introduction}

Diabetes mellitus (DM) is a major public health problem world-wide and is a significant health care challenge of this century [1]. Globally, it was estimated that 425 million people live with diabetes with 5 million deaths annually and the total global health expenditure due to diabetes was estimated at 673 billion US dollars [2]. It is a metabolic endocrine disorder characterized by chronic hyperglycemia, with disturbances of carbohydrate, fat and protein metabolism due to deficiencies in insulin secretion, insulin action, or both. Type 2 diabetes mellitus (T2DM), which is used to be only seen in adults but can now be diagnosed at any age, even with children; because it may remain undetected for many years [3]. Usually there is a strong association with people leading sedentary life style and people who are obese. Other risk factors that could lead to the development of T2DM include: family history and history of gestational diabetes. The effects of T2DM include long-term damage, dysfunction and failure of organs. Complications of DM may affect the functioning of various organ systems, and account for much of the morbidity and mortality associated with this disease [4]. These chronic complications can be broadly classified into two major categories: vascular and non-vascular. Vascular complications are further divided according to whether they are microvascular (retinopathy, neuropathy, and nephropathy) or macrovascular (coronary artery disease [CAD], peripheral 
arterial disease [PAD], and cerebrovascular disease). Conditions such as gastro paresis, infections, and skin changes constitute the non-vascular complications of diabetes [5].

Currently, T2DM is managed by a combination of diet, exercise and pharmacological therapy [6]. In one trial, combination therapy using high doses of thiazolidinediones (TZD), sulfonylureas (SU), metformin, and insulin, was found to be associated with cardiovascular problems and hypoglycemia. Moreover, metformin and TZDs decrease insulin resistance and hepatic glucose output in patients with significant renal or cardiac dysfunction, both of which occur frequently in T2DM [7]. The majority of oral hypoglycemic agents (OHAs) are vulnerable to impaired awareness of hypoglycemia with consequent neuroglycopenia and adverse cardiovascular effects, dictating the need for particular caution with therapies that increase the risk of hypoglycemia [8]. A history of severe hypoglycemia in older T2DM patients has been associated with a greater risk of dementia, which increases with the number of hypoglycemic episodes [9]. In recent years, incretin mimetics and DPP-4 inhibitors are believed to have advantages over other anti-diabetic drugs. Novel effects have been found with incretin-based therapies, such as GLP-1 receptor agonists (exenatide $\&$ liraglutide in phase 3 trials) and DPP-4 inhibitors (sitagliptin \& vildagliptin), which are beneficial to patients and are not found with other antidiabetic treatments in T2DM [10]. Perhaps the most significant of these is the glucose-dependent nature of their insulinotropic effects, which means that incretin-based therapies mimic closely the physiologic insulin profile and are associated with very low rates of hypoglycemia. In addition to this key property, incretin-based therapies do not cause weight gain in T2DM patients [11].

In 2013, the top 10 countries with higher prevalence of diabetes are Tokelau (37.5\%), Federated States of Micronesia (35\%), Marshall Islands (34.9\%), Kiribati (28.8\%), Cook Islands (25.7\%), Vanuatu (24\%), Saudi Arabia (23.9\%), Nauru (23.3\%), Kuwait (23.1\%) and Qatar (22.9\%) [12]. DM is no longer rare in Africa but it is the region with the lower prevalence of diabetes (4.9\%), having Reunion (15.4\%), Seychelles (12.1\%) and Gabon (10.7\%) as the top three countries with higher prevalence and 10 out of 48 countries with prevalence of diabetes higher than the upper quartile (6.3\%) prevalence [5]. Diabetes is one of the diseases in Ethiopia that is not given due attention. But as time goes, because of change of life style and other causes that have not been clearly revealed, the number of patients in the country is tolling at an alarming rate. According to the 2011 report, the number of adults living with DM in Ethiopia was 3.5\% [12]. However, many scholars believe that the prevalence could be above this value had it not been due to poor data collection and surveillance of the disease. Evidence shows that DM is claiming the lives of more than 4 million people worldwide annually and developing countries account for a substantially high proportion [13]. The prevalence of T2DM is rapidly increasing worldwide.

\section{Materials and Methods}

\subsection{Study Design and Sample Size}

A hospital based cross-sectional study was conducted in Tikur Anbessa Specialized Hospital and Huleshet Private Higher Clinic, Addis Ababa, Ethiopia, from December 2015 to May 2016. The study population consisted of 90 T2DM patients i.e., 60 patients who were receiving non incretin based therapy and 30 patients who were receiving incretin based therapy.

\subsection{Ethical Considerations}

Ethical clearance was obtained from the Ethical Committees of the Departments of Biochemistry and Internal Medicine, College of Health Sciences, Addis Ababa University. A consent form was prepared with a detailed explanation of the purpose, objectives, risks, and benefits of the study. Only those who agreed to participate were involved in the study. Sample collection was performed by trained health professionals following standard and ethical procedures.

\subsection{Sample Collection and Handling}

Collection of patients' data such as demographic characteristics, medical history, and the type of medication used as well as interview of professionals regarding T2DM management were carried out by the consulting physician. Venous blood $(5 \mathrm{ml})$ was drawn from each volunteer patient using a disposable plastic syringe. The blood was poured into a test tube and then centrifuged at 3500 revolution per minute for 10 minutes after it clotted. The serum was transferred to sterile tubes and stored in a deep freezer at $-70^{\circ} \mathrm{C}$ until analysis.

\subsection{Data Collection and Analyses of Samples}

Patient's weight and height were measured using standard weighing balance with height measuring device attached to the balance. Body Mass Index (BMI) was calculated from the body weight $(\mathrm{kg})$ and height $(\mathrm{m})$ as $\mathrm{BMI}=$ Weight $(\mathrm{kg}) /$ Height $\left(\mathrm{m}^{2}\right)$. Based on the National Institutes of Health Guidelines subjects were classified as follows: underweight if $\mathrm{BMI}<18.5 \mathrm{~kg} / \mathrm{m}^{2}$, normal if BMI is $18.5 \mathrm{~kg} / \mathrm{m}^{2}$ to 24.9 $\mathrm{kg} / \mathrm{m}^{2}$, overweight if BMI is 25.0 to $29.9 \mathrm{~kg} / \mathrm{m}^{2}$, and obese when BMI is $>30.0 \mathrm{~kg} / \mathrm{cm}^{2}$. Obesity is considered as one of the major predisposing factor to type 2 Diabetes [14]. Fasting Glucose levels in the patients' serum were estimated by using a commercial kit based on the method developed by Coxon and Schaffer obtained from Fluitest ${ }^{\circledR}$ GLU, Germany [15]. Serum total cholesterol was estimated by using a commercial kit, based on the method developed by Coxon \& Schaffer. Serum triglycerides and HDL-c level were estimated by using a commercial kit, based on the method developed by obtained from Cromatest ${ }^{\circledR}$ Cholesterol MR, Linear chemicals SL, Barcelona, Spain [16]. A method developed by Friedwald's was used to estimate LDL-c level in the serum samples [17]. 


\section{Results}

\subsection{Demographic and Anthropometric Results}

Among the 90 diabetic patients, 47(52.2\%) were Female and $43(47.8 \%)$ were Male. Out of the 60 diabetic individuals who received non-incretin based therapies $37(61.7 \%)$ were Female and 23(38.3\%) were Male and out of the 30 individuals who received incretin based therapies $10(33.3 \%)$ were Female and 20(66.7\%) were Male. The average age of diabetic patients who received non-incretin based therapies was $56.48 \pm 9.75$ years, ranging between 35 and 78 years. The average age of diabetic patients who received incretin based therapies was $56.50 \pm 9.77$ years, ranging between 32 and 72 years. The mean duration of diabetes for those who received non-incretin based therapies was $14.92 \pm 6.25$ years, ranging from 3 to 31 years, but for those who received incretin based therapies was $9.627 \pm 4.56$ years, ranging from 3 to 22 years. In the group who received non-incretin based therapies, 4 (6.7\%) drink alcohol but 56 (93.3\%) do not and in the group receiving incretin based therapies $8(27.7 \%)$ drink alcohol and $22(73.3 \%)$ do not. The patients in the nonincretin based group are nonsmokers but among patients receiving incretin based therapy $5(27.7 \%)$ are smokers and $25(73.3 \%)$ are nonsmokers. In the group who received nonincretin based therapies $10(17.7 \%)$ had family history of diabetes and $50(83.3 \%)$ had no family history but among diabetic patients who received incretin based therapies 5 $(17.7 \%)$ had family history of diabetes and $25(83.3 \%)$ had no family history. Regarding the calculated BMI, the minimum value was $15.80 \mathrm{~kg} / \mathrm{m}^{2}$ and the maximum was $40.80 \mathrm{~kg} / \mathrm{m}^{2}$. In the nonincretin based treated group, 21 (35.00\%) had normal body mass index, $23(38.30 \%)$ were overweight, $13(21.70 \%)$ were obese and $3(5.00 \%)$ were underweight. However, in the incretin based treated group 7 $(23.30 \%)$ had normal BMI, 1(3.30\%) was overweight, 22 $(73.30 \%)$ were obese and none of the study participants were found to be underweight (Table 1).

Table 1. Socio- demographic characteristics of diabetic patients in the study.

\begin{tabular}{lll}
\hline Variable & Non-incretin based treated Number (\%) & Incretin based treated Number (\%) \\
\hline Sex & $37(61.7)$ & $10(33.3)$ \\
Male & $23(38.3)$ & $20(66.7)$ \\
Female & & \\
Age in years & $0(0)$ & $0(0)$ \\
$18-30$ & $5(8.3)$ & $1(3.3)$ \\
$31-40$ & $10(16.7)$ & $9(30.0)$ \\
$41-50$ & $24(40.0)$ & $10(33.3)$ \\
$51-60$ & $21(35.0)$ & $10(33.3)$ \\
$>60$ & & \\
Duration of diabetes (Years) & $1(1.7)$ & $2(6.7)$ \\
$<5$ & $20(33.3)$ & $20(66.7)$ \\
$5-10$ & $39(65.0)$ & $8(26.7)$ \\
$>10$ & $10(17.7)$ & $5(17.7)$ \\
Family history of diabetes & $50(83.3)$ & $25(83.3)$ \\
Yes & & $5(17.7)$ \\
No & $0(0)$ & $25(83.3)$ \\
Smoking status & $60(100)$ & $8(27.7)$ \\
Yes & & $22(73.3)$ \\
No & $4(6.7)$ & $0(0)$ \\
Alcohol drinking & $56(93.3)$ & $7(23.3)$ \\
Yes & $3(5)$ & $1(3.3)$ \\
No & $21(35.0)$ & $22(73.3)$ \\
BMI $\left(\right.$ Kg/m $\left.{ }^{2}\right)$ & $23(38.3)$ & \\
$<18.5$ & $13(21.7)$ & \\
$18.5-24.9$ & & \\
$25-29.9$ & & \\
$>30$ & &
\end{tabular}

\subsection{Biochemical Related Results}

The mean values of lipid profile, FBS, renal function, and BMI of all diabetic patients from base line is shown in Table
2 and there were no significant differences in both groups. The TC values and duration of stay with diabetes were significantly higher in the non-incretin based treated group than the incretin based treated group $(\mathrm{P}=0.013$ and $\mathrm{P}=0.001)$.

Table 2. The baseline characteristics of all patients at randomization.

\begin{tabular}{llll}
\hline Biochemical parameters & Incretin treated & Non-incretin treated & P value \\
\hline Sex: Male/Female & $10 / 20$ & $37 / 23$ & - \\
Age (years) & $56.5 \pm 9.7$ & $56.1 \pm 10.1$ & 0.847 \\
Diabetes duration (years) & $9.62 \pm 4.5$ & $14.92 \pm 6.25$ & $0.001^{*}$ \\
FBS (mg/dl) & $188.08 \pm 78.74$ & $171.73 \pm 72.72$ & 0.331 \\
\hline
\end{tabular}




\begin{tabular}{llll}
\hline Biochemical parameters & Incretin treated & Non-incretin treated & P value \\
\hline TC $(\mathrm{mg} / \mathrm{dl})$ & $171.87 \pm 49.82$ & $196.65 \pm 40.38$ & $0.013^{*}$ \\
TG $(\mathrm{mg} / \mathrm{dl})$ & $184.23 \pm 53.74$ & $185.60 \pm 71.60$ & 0.927 \\
LDL $(\mathrm{mg} / \mathrm{dl})$ & $115.33 \pm 37.92$ & $125.88 \pm 39.39$ & $0.227^{*}$ \\
HDL $(\mathrm{mg} / \mathrm{dl})$ & $35.83 \pm 13.14$ & $36.12 \pm 7.92$ & 0.899 \\
Urea $(\mathrm{mg} / \mathrm{dl})$ & $26.53 \pm 8.27$ & $26.50 \pm 8.34$ & 0.986 \\
Creatinine $(\mathrm{mg} / \mathrm{dl})$ & $0.94 \pm 0.12$ & $0.956 \pm 0.12$ & 0.826 \\
BMI $\left(\mathrm{kg} / \mathrm{m}^{2}\right)$ & $25.35 \pm 3.05$ & $25.04 \pm 3.98$ & 0.800 \\
\hline
\end{tabular}

*The mean is significant at $\mathrm{p}<0.05$ (By using independent $\mathrm{t}$ test analysis).

The percentages of patients taking different anti-diabetic drugs were different within the study group. Eleven types of anti-diabetes drugs were used in the clinics during the study period and they were used either as single or combination therapy. Majority of the diabetic patients who received nonincretin based therapies were treated with metformin and Neutral Protamine Hagedorn (NPH) 29 (32.2\%) as combination therapy and majority of the patients who received incretin-based therapy were treated with Galvusmet 11(12.2\%). Majority of the diabetic patients who received non-insulin based therapies were treated with metformin $14(15.6 \%)$ and majority of the patients who received incretin-based therapy were treated with Onglyza $7(7.8 \%)$ among the groups on single therapy. The doses of the drugs were metformin $(500-2000 \mathrm{mg} /$ day), glibenclamide (2.5$20 \mathrm{mg} /$ day), and various units of insulin. The concentration of the drugs given to patients on incretin-based therapy group were Sitagliptin (Januvia) with $25 \mathrm{mg}, 50 \mathrm{mg}, 100 \mathrm{mg}$ tablets and also in combination with metformin (Janumet) with $50 / 500 \mathrm{mg}$, Vildagliptin (Galvus) with 50mg tablets and also in combination with metformin (Galvusmet) with 50/500mg, Saxagliptin (Onglyza) with $5 \mathrm{mg}$ tablets and also in combination with metformin (Konbolyza) with 5/500mg (Table 3).

Table 3. Percentage of Anti-diabetic Medications prescribed to patients during the study period.

\begin{tabular}{|c|c|c|c|}
\hline Type of therapy & Drugs & Freequency & Percentage \\
\hline \multirow[t]{4}{*}{ Non-incretin based } & Metformin & 14 & 15.6 \\
\hline & Metformin + NPH & 29 & 32.2 \\
\hline & Glibencamide + Metformin & 4 & 4.4 \\
\hline & Glibencamide $+\mathrm{NPH}$ & 6 & 6.7 \\
\hline \multirow[t]{5}{*}{ Incretin based } & Januvi & 4 & 4.4 \\
\hline & Janumet & 2 & 2.2 \\
\hline & Galvusmet & 11 & 12.2 \\
\hline & Onglyza & 7 & 7.8 \\
\hline & Konbolyza & 1 & 1.1 \\
\hline TOTAL & & 90 & 100 \\
\hline
\end{tabular}

The mean FBS level of diabetic patients who received incretin-based therapy $(152.87 \pm 51.977 \mathrm{mg} / \mathrm{dl})$ was lower than that of diabetic patients who received non-incretin based therapy $(189.34+81.520 \mathrm{mg} / \mathrm{dl})$ as shown in Table 4 . The mean value of lipid profiles (TC, TG, and LDL) in diabetic patients who received incretin-based therapy was significantly lower than the mean value of diabetic patients who received non-incretin based therapy $(\mathrm{p}=0.028, \mathrm{P}=0.000$, and $\mathrm{P}=0.002)$ respectively and higher mean level of HDL $(\mathrm{p}=0.003)$ as shown in Table 4. The mean serum creatinine and urea levels of diabetic patients who received incretin-based therapies were significantly $(p<0.5)$ lower compared to that of diabetic patients who received noninsulin based therapies (Table 4). The mean BMI of diabetic patients who received incretin-based therapies was also significantly lower compared to that of diabetic patients who received non-insulin based therapies.

Table 4. Mean $\pm S D$ of serum lipid profile, renal function test values, BMI and FBS level of non-incretin and incretin based treated diabetic patients.

\begin{tabular}{llll}
\hline Parameters & Incretin-based $(\mathbf{N}=\mathbf{3 0})$ & Non-incretin based $(\mathbf{N}=\mathbf{6 0})$ & P-value \\
\hline FBS $(\mathrm{mg} / \mathrm{dl})$ & $152.87 \pm 51.98$ & $189.34 \pm 81.52$ & $0.028^{*}$ \\
TC 9mg/dl) & $142.47 \pm 41.75$ & $188.25 \pm 50.50$ & $0.001^{*}$ \\
TG 9 mg/dl) & $147.70 \pm 74.03$ & $160.50 \pm 90.26$ & 0.594 \\
LDL (mg/dl) & $88.47 \pm 31.84$ & $116.33 \pm 42.70$ & $0.002^{*}$ \\
HDL (mg/dl) & $44.77 \pm 10.43$ & $36.67 \pm 12.36$ & $0.003^{*}$ \\
Urea (mg/dl) & $17.20 \pm 4.57$ & $34.03 \pm 9.15$ & $0.001^{*}$ \\
Creatinine (mg/dl) & $0.707 \pm 0.33$ & $0.989 \pm 0.18$ & $0.006^{*}$ \\
BMI $\left(\mathrm{kg} / \mathrm{m}^{2}\right)$ & $24.1 \pm 3.63$ & $26.6 \pm 4.21$ & 0.108 \\
\hline
\end{tabular}

Mean $\pm \mathrm{SD}$ is significant at $p<0.05$ (Independent Samples Test), FBS =fasting blood glucose, TC $=$ total cholesterol, HDL $=$ high density lipoprotein, $\mathrm{LDL}=$ low density lipoprotein, BMI=body mass index.

After treatment, the mean FBS values of incretin-based treated diabetic patients was found to be reduced by $35.21 \pm$
$26.77 \mathrm{mg} / \mathrm{dl}$ and the mean BMI value was also reduced by $1.25 \mathrm{~kg}+0.58$ from baseline. This difference was not 
statistically significant $(\mathrm{p}>0.05)$. The mean lipid profile (TC, TG, LDL and HDL) and renal function test (serum urea and serum creatinine) values of incretin-based treated diabetic patients showed a statistically significant reduction from baseline $(\mathrm{p}<0.05)$ as shown in Table 5 .

Table 5. Comparison of Mean \pm SD of serum lipid profile, renal function test, FBS and BMI of incretin based treated diabetic patients from baseline.

\begin{tabular}{llll}
\hline Biochemical Parameters & Incretin based treated & P-Value \\
\hline & Before & Final & 0.050 \\
FBS $(\mathrm{mg} / \mathrm{dl})$ & $188.08 \pm 78.743$ & $152.87 \pm 51.977$ & $0.015^{*}$ \\
TC $(\mathrm{mg} / \mathrm{dl})$ & $171.87 \pm 49.821$ & $142.47 \pm 41.755$ & $0.021^{*}$ \\
TG $(\mathrm{mg} / \mathrm{dl})$ & $184.23 \pm 53.742$ & $147.70 \pm 74.026$ & $0.006^{*}$ \\
LDL (mg/dl) & $115.33 \pm 37.921$ & $88.47 \pm 31.837$ & $0.015^{*}$ \\
HDL (mg/dl) & $35.83 \pm 13.143$ & $44.77 \pm 10.434$ & $0.001^{*}$ \\
Urea $(\mathrm{mg} / \mathrm{dl})$ & $26.53 \pm 8.274$ & $17.20 \pm 4.567$ & $0.012^{*}$ \\
Creatinine (mg/dl) & $0.94 \pm 0.117$ & $0.707 \pm 0.326$ & 0.232 \\
BMI $\left(\mathrm{Kg} / \mathrm{m}^{2}\right)$ & $25.35 \pm 3.05$ & $24.1 \pm 3.63$ & \\
\hline
\end{tabular}

${ }^{*}$ Mean $\pm \mathrm{SD}$ is significant at $=p<0.05$ (by Paired t- test)

The mean FBS values in case of non-insulin based treated diabetic patients was found to be increased by $17.61 \pm 8.8$ $\mathrm{mg} / \mathrm{dl}$ and the mean BMI value also increased by $1.56 \mathrm{~kg} \pm 0.23$ from baseline but not significantly $(p>0.05)$. The mean lipid profile (TC, TG and LDL) values were reduced from baseline and mean value level of HDL non-significantly increased from baseline $(\mathrm{p}>0.05)$. The mean serum urea value level were significantly increased $(p<0.05)$ from base line and the mean value serum creatinine level was increased but not significant $(\mathrm{p}>0.05)$ from base line as shown in Table 6.

Table 6. Comparison of Mean $\pm S D$ of serum lipid profile, renal function test, FBS and BMI of all diabetics with non-insulin based treated diabetic patients from base line.

\begin{tabular}{llll}
\hline Biocheical Parameters & Non-incretin based treated & P-Value & Final \\
\hline & Before & & 0.236 \\
FBS $(\mathrm{mg} / \mathrm{dl})$ & $171.73 \pm 72.720$ & $189.34 \pm 81.520$ & 0.352 \\
TC $(\mathrm{mg} / \mathrm{dl})$ & $196.65 \pm 40.385$ & $188.25 \pm 50.501$ & 0.111 \\
TG $(\mathrm{mg} / \mathrm{dl})$ & $185.60 \pm 71.603$ & $160.50 \pm 90.264$ & 0.244 \\
LDL $(\mathrm{mg} / \mathrm{dl})$ & $125.88 \pm 39.398$ & $116.33 \pm 42.698$ & 0.787 \\
HDL $(\mathrm{mg} / \mathrm{dl})$ & $36.12 \pm 7.925$ & $36.67 \pm 12.361$ & $0.001^{*}$ \\
Urea $(\mathrm{mg} / \mathrm{dl})$ & $26.50 \pm 8.345$ & $34.03 \pm 9.154$ & 0.732 \\
Creatinine $(\mathrm{mg} / \mathrm{dl})$ & $0.956 \pm 0.118$ & $0.989 \pm 0.181$ & 0.502 \\
BMI $\left(\mathrm{Kg} / \mathrm{m}^{2}\right)$ & $25.04 \pm 3.98$ & $26.6 \pm 4.21$ & \\
\hline
\end{tabular}

*Mean $\pm \mathrm{SD}$ is significant at the $p<0.05$ (by Paired t- test).

The mean values of FBS, BMI, TC, TG, LDL, and Serum urea were increased in the female as compared to male patients in both groups and the female had low HDL and serum creatinine as compared to the male (Table 7).
However, the mean difference of female and male diabetic patient of FBS, lipid profiles and serum urea were not statistically significant $(\mathrm{p}>0.05)$.

Table 7. Comparison of Mean + SD of serum lipid profile, renal function tests, FBS and BMI of all incretin and non-incretin based treated diabetic patients based on gender.

\begin{tabular}{|c|c|c|c|c|c|}
\hline Parameters & Sex & No. & Mean & Std. deviation & P-Value \\
\hline \multirow[t]{2}{*}{ FBS (mg/dl) } & Female & 47 & 187.71 & 69.366 & 0.164 \\
\hline & Male & 43 & 165.67 & 79.402 & \\
\hline \multirow[t]{2}{*}{$\mathrm{TC}(\mathrm{mg} / \mathrm{dl})$} & Female & 47 & 177.53 & 52.345 & 0.391 \\
\hline & Male & 43 & 168.02 & 52.300 & \\
\hline \multirow[t]{2}{*}{$\mathrm{TG}(\mathrm{mg} / \mathrm{dl})$} & Female & 47 & 166.79 & 86.843 & 0.220 \\
\hline & Male & 43 & 144.70 & 82.378 & \\
\hline \multirow[t]{2}{*}{$\mathrm{LDL}(\mathrm{mg} / \mathrm{dl})$} & Female & 47 & 110.72 & 40.539 & 0.381 \\
\hline & Male & 43 & 103.02 & 42.415 & \\
\hline \multirow[t]{2}{*}{ HDL (mg/dl) } & Female & 47 & 38.98 & 12.229 & 0.757 \\
\hline & Male & 43 & 39.79 & 12.527 & \\
\hline \multirow[t]{2}{*}{ Urea $(\mathrm{mg} / \mathrm{dl})$} & Female & 47 & 29.21 & 10.691 & 0.488 \\
\hline & Male & 43 & 27.56 & 11.849 & \\
\hline \multirow[t]{2}{*}{ Creatinine $(\mathrm{mg} / \mathrm{dl})$} & Female & 47 & 0.651 & 0.2781 & $0.001^{*}$ \\
\hline & Male & 43 & 0.893 & 0.2667 & \\
\hline \multirow[t]{2}{*}{ BMI $\left(\mathrm{Kg} / \mathrm{m}^{2}\right)$} & Female & 47 & 25.938 & 4.3413 & $0.030^{*}$ \\
\hline & Male & 43 & 24.156 & 3.1964 & \\
\hline
\end{tabular}


Variable factors like age, BMI and duration of diabetes which may affect lipid profiles, renal function tests, and FBS level were correlated. BMI correlated positively with the levels of TC $(r=0.154)$, TG $(r=0.162)$, LDL-c $(r=0.077)$ and negatively with HDL-c $(r=0.148)$ but the correlation was statistically not significant $(\mathrm{p}>0.05)$ as shown in Figure 1 . The BMI value was also correlated positively with serum urea level $(\mathrm{r}=0.023)$, FBS level $(\mathrm{r}=0.099)$ and correlated negatively with serum creatinine level $(\mathrm{r}=0.099)$ but statistically not significant $(\mathrm{p}>0.05)$. Further correlation studies were conducted between FBS, TC, TG, LDL-c, HDLc, serum urea and creatinine level with duration of diabetes. A positive correlation was obtained with the level of TC $(\mathrm{r}=0.247)$, TG $(\mathrm{r}=0.139)$, LDL-c $(\mathrm{r}=0.251)$, serum urea $(\mathrm{r}=0.369)$, FBS $(\mathrm{r}=0.219)$ and negative correlation with the level of HDL-c $(r=0.056)$ and serum creatinine $(r=0.167)$. But, there was significant moderate positive correlation between TC, LDL-c, serum urea and FBS $(\mathrm{p}<0.05)$ respectively as shown in Figures 2, 3, and 4.

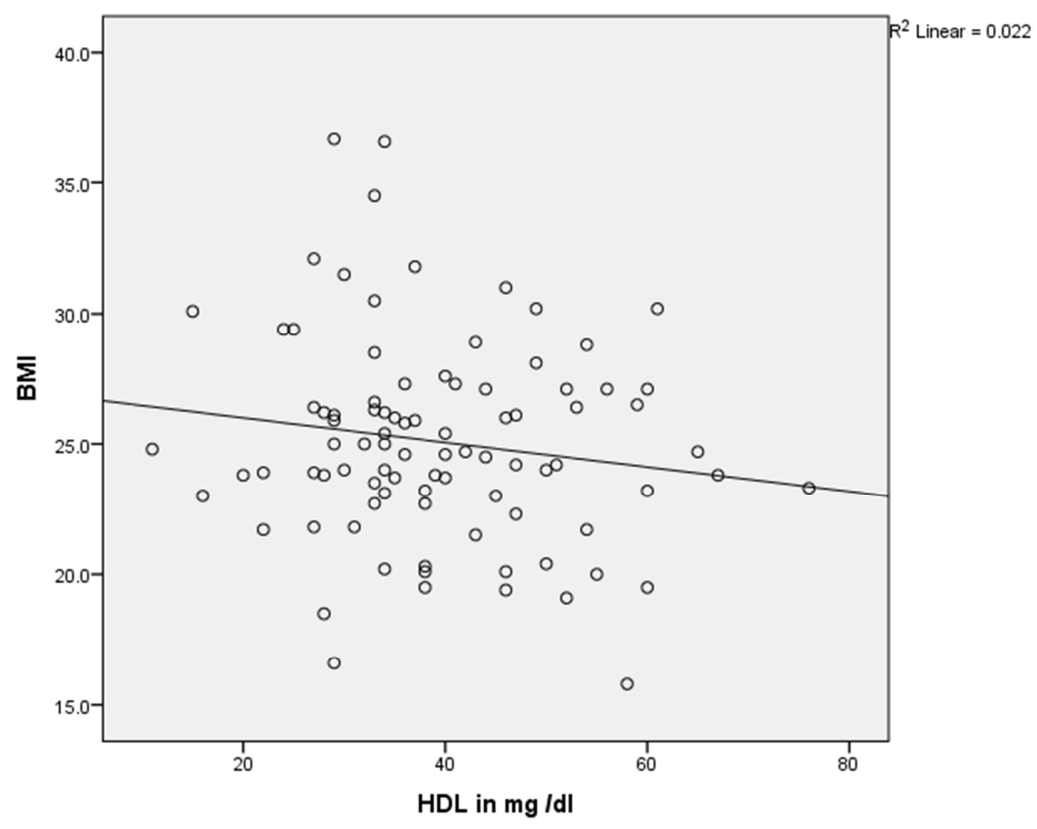

Figure 1. Correlation between BMI and HDL-c levels.

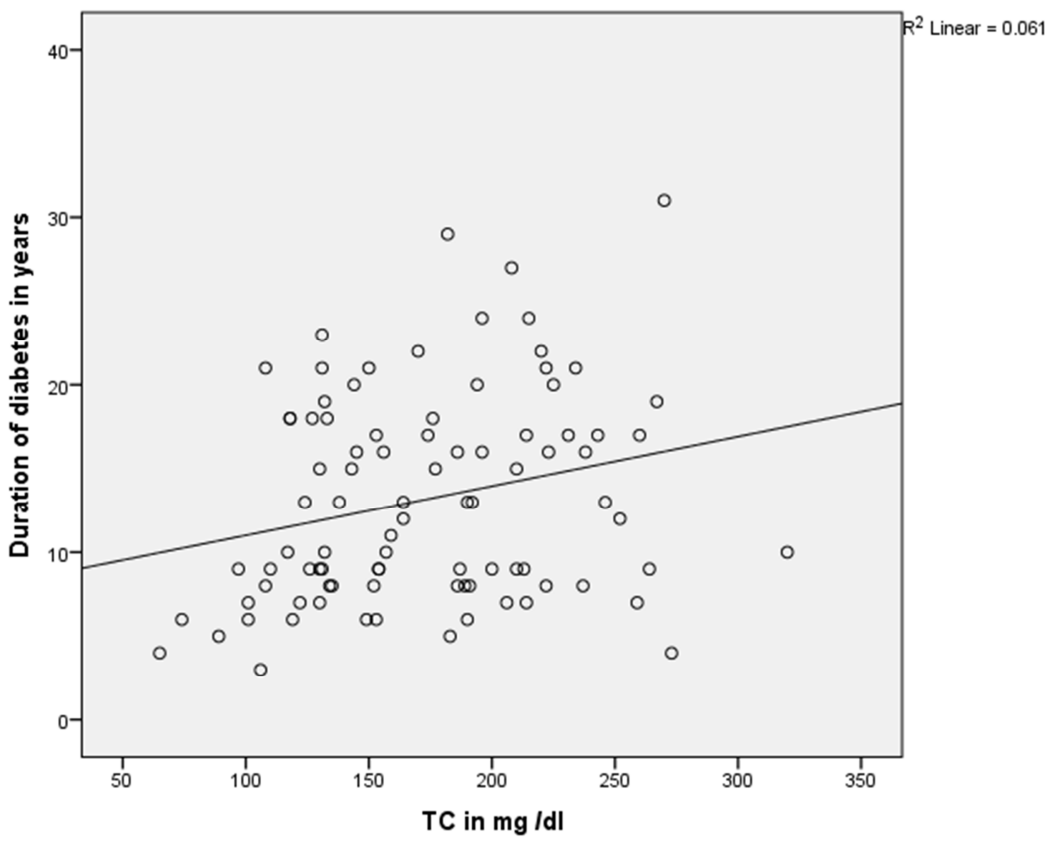

Figure 2. Correlation between duration of diabetes mellitus and TC levels. 


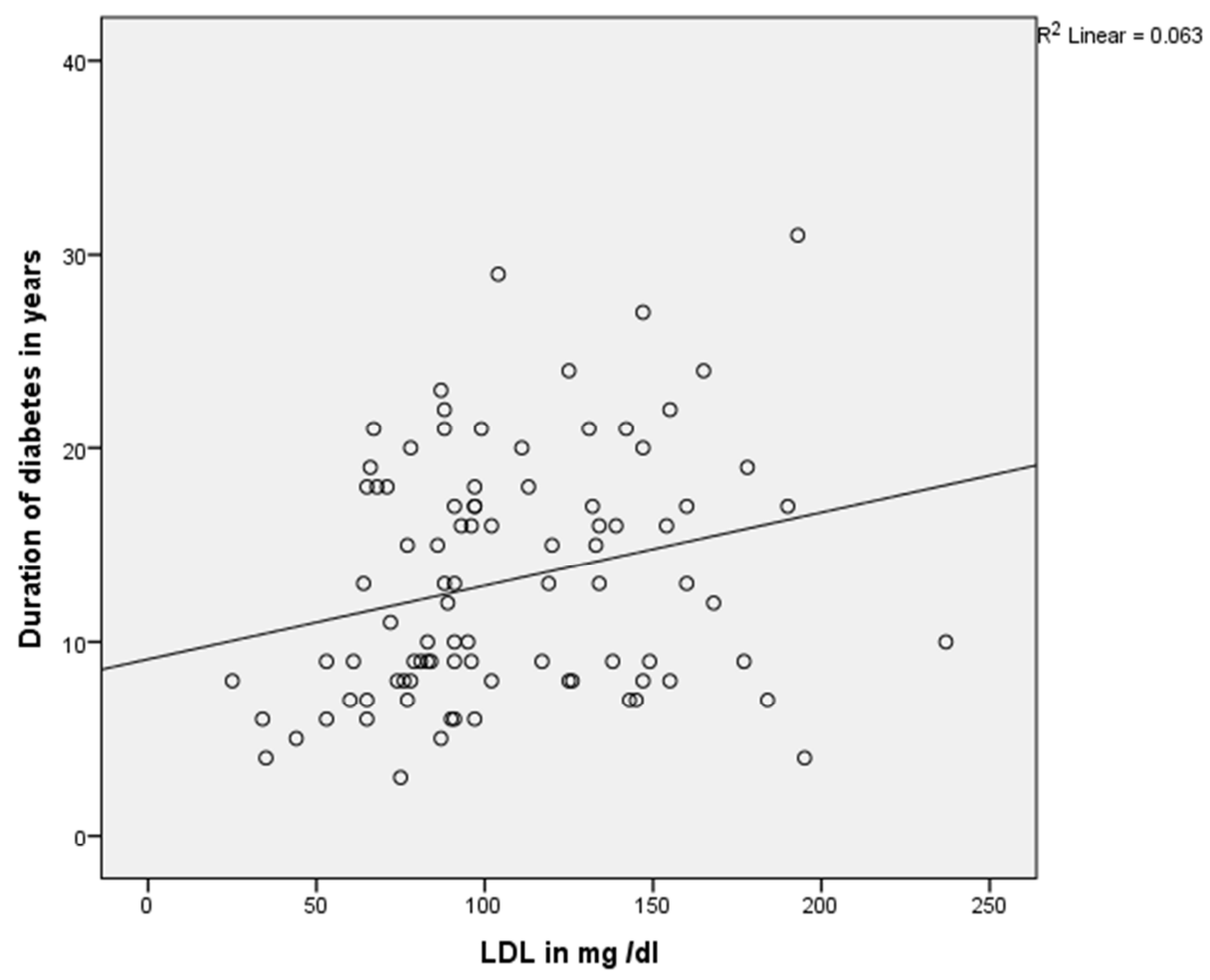

Figure 3. Correlation between duration of diabetes and $L D L-c$ levels.

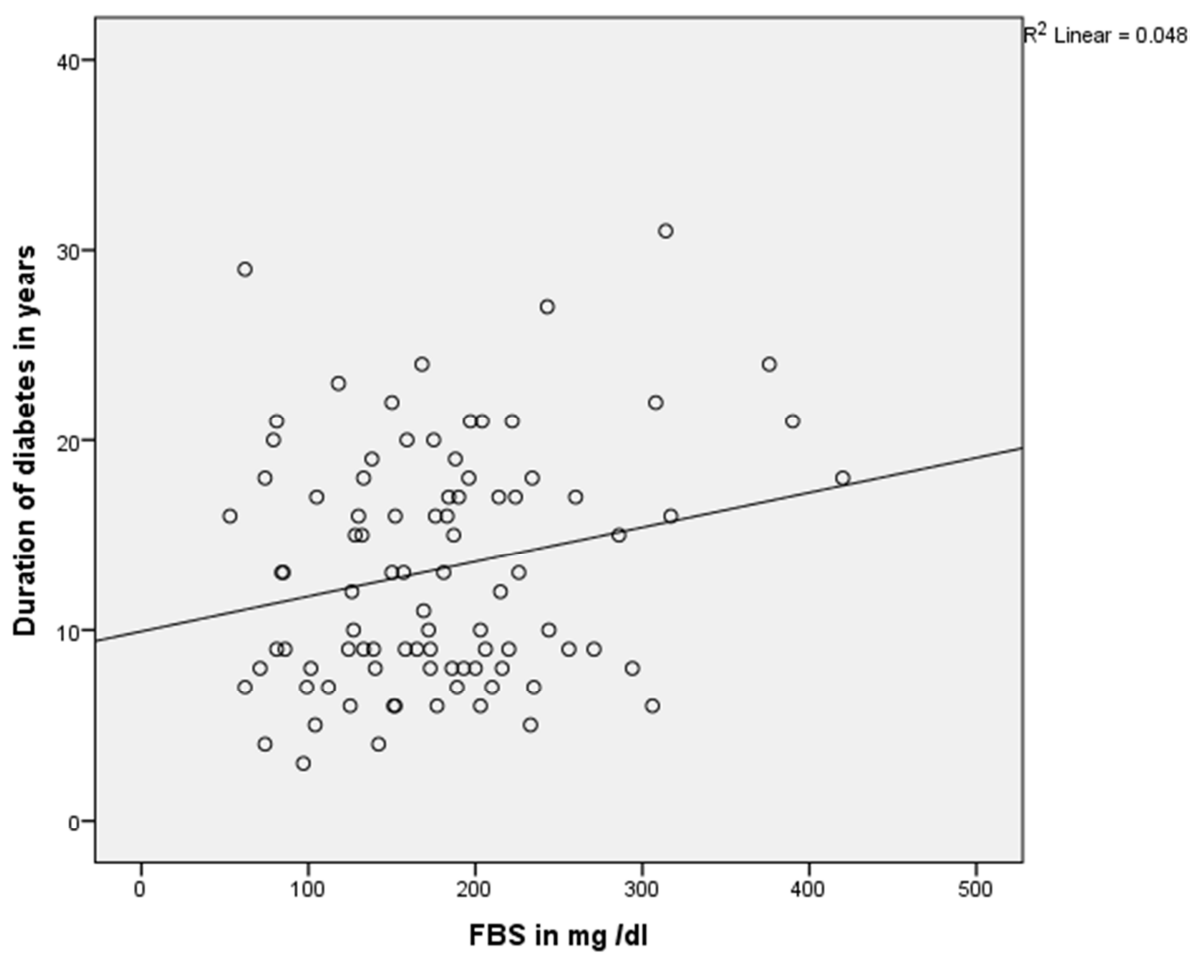

Figure 4. Correlation between duration of diabetes mellitus and FBS levels.

\section{Discussion}

The growing epidemic of T2DM and the recognition that achieving specific glycemic goals can substantially reduce morbidity have made the effective treatment of hyperglycemia a top priority. Current T2DM treatment regimens do not effectively target the fundamental defects in glucose-mediated insulin secretion and beta-cell loss; hence as a result, an increasing proportion of T2DM patients are believed to progress to requiring insulin [18]. A majority of oral hypoglycemic agents in common use are insulin 
sensitizers and/or insulin secretagogues (insulin release pills). In recent years incretin based therapies have come to the forefront as alternative strategies for treatment of T2DM. The incretin hormones i.e., glucagon like peptide-1 (GLP-1) and glucose dependent insulinotropic peptide (GIP) have the potential to address these defects which are implicated in presenting a major paradigm shift in the management of T2DM patients. The actions of incretins improve the defects in glucose metabolism, pancreatic function and energy production in T2DM patients [19]. These findings have led to the development of once-daily, orally-active DPP-4 inhibitors to increase the incretin effect. DPP -IV inhibitors have good tolerability and are associated with very few side effects. There are no long-term data on the effects of DPP-4 inhibitors on diabetes-related complications and mortality. To our knowledge, no clinical studies have been specifically made to show the relationship between incretin-based therapies and the non-insulin based therapies among T2DM on lipid profile and renal function test values since it's recently introduced in Ethiopia as a treatment strategy. Therefore, this study was done to unravel which of the strategies better reverse glycemic conditions and also improve lipid profiles in order to avoid diabetic complications. The results regarding plasma glucose indicate that there was an improvement after treatment courses of one year with incretin-based therapies' particularly the oral administration of the DPP-4 inhibitors either as mono therapy or combination with metformin as compared to that of diabetic patients who have received non-insulin based therapies either as mono or combination therapy. The FBS level of diabetic patients who were treated with incretinbased therapies was lower than that of diabetic patients who were treated with non-insulin based therapies and the reversal effect was very significant. This could be because such treatments may stimulate insulin secretion, reduce gastric emptying thereby improving appetite and also inhibit glucagon secretion. A similar result was obtained by other researchers [20, 21]. However, despite the fact that these patients were having follow-ups in the two diabetic clinics, their blood sugar level was still in the hyperglycemic range showing they had poor glycemic control. The reason for this could be multifaceted: one reason could be that patients do not adhere to their regiments or they don't observe the exact time of taking their drugs; or it could be that they did not have a properly scheduled follow up to scale up their doses. Lipid profile plays an important role in cardiovascular risk assessment. One of the major risk factors for the development of cardiovascular disease is dyslipidemia, which may be primary or associated with hypertension, diabetes mellitus and obesity. Findings of this study indicated that incretin-based therapy also improves the lipid profiles of type 2 diabetic patients better than the patients that were under non-insulin based treatment. When the data for lipid profiles of incretin-based treated diabetic patients were compared with that of non-insulin based treated diabetic patients the former treatment strategies showed a better adjustment of lipid profiles to baseline values. Although, concerns regarding cardiovascular safety after use of DPP-4 inhibitors are persisting, many studies did not show any increased risk in type-2 diabetic management, after treating with DPP-4 inhibitors [22-24]. The clinical practice guidelines and use of oral antihyperglycaemic agents for adult patients must be up-dated with type-2 diabetes for better results similar to the Korean Diabetes Association [25].

T2DM patients receiving DPP-4 inhibitors also seem to protect patients from developing kidney problems as observed through measurement of creatinine and urea. No drug-related serious adverse events were reported after six months of DPP-4 inhibitor based combination therapies. Similar studies had suggested that sitagliptin may be used as monotherapy in patients who cannot tolerate metformin or sulfonylurea, and sitagliptin may be used as alternative to metformin in cases with renal insufficiency [26]. This study is preliminary as it was done on small number of patients and the effect of the drugs on cancer development as well as their effect on ketone production was not investigated. Hence a broader and protracted study involving a wider range of parameters and spectrum of the effect and efficacy of these treatment strategies must be done.

\section{Conclusion}

In conclusion, we have demonstrated a oral glycemic control mechanism, in type-2 diabetic patients using incretinbased therapies in an Ethiopian referral hospital and a primary care facility in Addis Ababa, Ethiopia. This likely represents a controllable burden of alleviating hyperglycemic situations in diabetic patients who are on combination therapy/metformin add-on therapy and who are inadequately controlled by metformin and sulphonylureas. This work shows that DPP-4 inhibitors based therapy for treatment of T2DM either as mono-therapy or in combination therapy corrects lipid profile, renal function values, fasting blood sugar and weight gain as compared to non-insulin based therapies either as mono-therapy or in combination for treatment of T2DM. Generally, DPP-4 inhibitors based therapy showed clinically significant improvement in lipid profile correction and renal function. DPP-4 inhibitors based therapy administration is particularly beneficial in overweight and obese patients who represent the majority of patients with T2DM, as well as in elderly patients and in diabetics who are susceptible to hypoglycemia. Furthermore, routine biochemical tests should be performed on serum samples of diabetic patients, in order to assess for risk factors of hypoglycemia, kidney failure and cancer in these highburdened settings.

\section{Data Availability}

All the data of this work is available as repository of Addis Ababa University Library, which can be visited at www.aau.edu.et. 


\section{Conflict of Interest}

The authors of this manuscript do not have any conflict of interest what so ever.

\section{Funding Statement}

This work was done through an MSC project research fund released by Addis Ababa University to Alias as partial fulfillment and the university was not involved $\mathrm{n}$ the preparation of this manuscript.

\section{Acknowledgement}

Authors thank Addis Ababa University and Huleshet Higher Clinic for their support. Authors also greatly acknowledge all patients who were willing to participate in this study.

\section{References}

[1] Rudasingwa GJ, Amendezo E, Twagirumukiza M (2012) Clinical patterns and complications of African diabetes patients: preliminary data from Kigali University Teaching Hospital, Rwanda. Afr. J Diab Med 20: 39-42.

[2] Ogurtsova K, da Rocha Fernandes JD, Huang Y, Linnenkamp U, Guariguata L, Cho NH, Cavan D, Shaw JE, Makaroff LE (2017) IDF Diabetes Atlas: Global estimates for the prevalence of diabetes for 2015 and 2040. Diabetes Res Clin Pract 128: 40-5.

[3] American Diabetic Assiciation (2009) Diagnosis and Classification of Diabetes Mellitus. Diabetes Care 32 (Suppl 1): S62-S67.

[4] Anjali DD, Marcie HH, Mario S (2008) Epidemiology of Diabetes and Diabetes-Related Complications. Phys Ther 88(11): 1254-126.

[5] International Diabetes Federation (2013) Atlas of Diabetes; sixth edition, $\mathrm{p} 7$.

[6] Fowler MJ (2008) Microvascular and macrovascular complications of diabetes. Clinical diabetes, 26(2): 77-82.

[7] Action to Control Cardiovascular Risk in Diabetes Study Group (2008) Effects of intensive glucose lowering in type 2 diabetes. The New Engl. J. Med. 358(24):2545.

[8] Holstein A, Egberts EH (2003) Risk of hypoglycaemia with oral antidiabetic agents in patients with Type 2 diabetes. Exp Clin Endocrinol Diabetes 111(7): 405-14.

[9] Whitmer RA, Karter AJ, Yaffe K, Quesenberry CP, Selby JV (2009). Hypoglycemic episodes and risk of dementia in older patients with type 2 diabetes mellitus. JAMA 301(15): 15651572 .

[10] Pratley RE, Salsali A (2007) Inhibition of DPP-4: a new therapeutic approach for the treatment of type 2 diabetes. Curr Med Res opin 23(4): 919-931.

[11] Nauck MA, Vardarli I, Deacon CF, Holst JJ, Meier JJ (2011) Secretion of glucagon-like peptide-1 (GLP-1) in type 2 diabetes: what is up, what is down? Diabetologia 54(1): 10-18.
[12] International Diabetes Federation (2011) Atlas of Diabetes global estimates of the Prevalence of diabetes for 2011 and 2030 .

[13] Wild S, Roglic G, Green A, Sicree R, King H (2004) Global prevalence of diabetes estimates for the year 2000 and projections for 2030. Diabetes care 27(5): 1047-1053

[14] Hao X, Ke-ji C (2011) Integrating traditional medicine with biomedicine towards a patient-centered healthcare system. Chin J Integ Med 17(2): 83-84.

[15] Coxon B, Schaffer R (1971) Characterization and quantitative analysis of D-glucose for use in clinical analysis. Analytical chemistry 43(12): 1565-1570.

[16] Allain, CC, Poon, LS, Chan, CS Richmond, WF, Fu, PC (1974). Enzymatic determination of total serum cholesterol. Clinical chemistry 20(4): 470-475.

[17] Friedewald, WT, Levy RI, Fredrickson DS (1972) Estimation of the concentration of low-density lipoprotein cholesterol in plasma, without use of the preparative ultracentrifuge. Clin Chem 18(6): 499-502.

[18] Marín-Peñalver JJ, Martín-Timón I, Sevillano-Collantes C, del Cañizo-Gómez FJ (2016) Update on the treatment of type 2 diabetes mellitus. World J Diab 7(17): 354-395.

[19] Khoo J, Rayner CK, Jones KL, Horowitz M (2009) Incretinbased therapies: new treatments for type 2 diabetes in the new millennium. Ther Clin Risk Manag 5(3): 683-698.

[20] Karen B, Mary E, Cox B (2010) Clinical utility of fixed combinations of sitagliptin-metformin in treatment of type 2 diabetes. Metab Synd Obes 3:363- 372.

[21] Shannon AM, Erin LO, Roger AJ (2009) Sitagliptin as combination therapy in the treatment of type 2 diabetes mellitus. Diabetes Metab Syndr Obes 2: 23-30.

[22] Green TB, Bethel MA, Armstrong PW et al (2015) Effect of Sitagliptin on cardiovascular outcomes in Type-2 diabetes. 2015. N. Engl. J. med. 373: 232-242. doi: 10. 1056/NE JMOa1501352.

[23] Liu J, Li L, Deng K, Xu C, Busse J et al (2017) Incretin based treatments and mortality in patients with type 2 diabetes: Systemic review and meta analysis. BMJ 357: j2499. doi: https//doi.org/10. 1136 bmj.j2499.

[24] Chin HJ, Nam JH, Lee EK, Shin J (2017) Comparative safety for cardiovascular outcomes of DPP-4 inhibitors versus glimepiride in patients with type-2 diabetes. Medicine 96(25): e7213. doi: 10. 1097/MD. 0000000000007213.

[25] Moon MK, Hur KY, Ko SH, Park SO, Lee BW et al (2017) Combination therapy of oral hypoglycemic agents in patients with type 2 diabetes mellitus. Diabetes Metab. J 41(5): 357366. doi: 10. 4093/dmj. 2017. 41. 5. 357.

[26] Chan JC, Scott R, Arjona Ferreira JC, Sheng D, Gonzalez E, et al (2008) Safety and efficacy of Sitagliptin in patients with type 2 diabetes and chronic renal insufficiency. Diabetes Obes Metab. 10(7): 545-555. doi: 10. 1111/j. 1463-1326. 2008. 00914.x. E Pub2008Jun1. 